\title{
BMJ Open NAITRE study on the impact of conditional cash transfer on poor pregnancy outcomes in underprivileged women: protocol for a nationwide pragmatic cluster-randomised superiority clinical trial in France
}

To cite: Bardou M, Crépon B, Bertaux A-C, et al. NAITRE study on the impact of conditional cash transfer on poor pregnancy outcomes in underprivileged women: protocol for a nationwide pragmatic clusterrandomised superiority clinical trial in France. BMJ Open 2017;7:e017321. doi:10.1136/ bmjopen-2017-017321

- Prepublication history and additional material for this paper are available online. To view these files, please visit the journal online (http://dx.doi. org/10.1136/bmjopen-2017017321).

Received 19 April 2017 Revised 15 September 2017 Accepted 27 September 2017

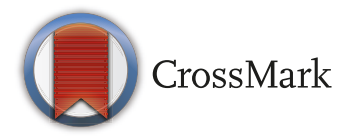

For numbered affiliations see end of article.

Correspondence to

Dr Marc Bardou;

marc.bardou@u-bourgogne.fr

\section{ABSTRACT}

Introduction Prenatal care is recommended during pregnancy to improve neonatal and maternal outcomes. Women of lower socioeconomic status (SES) are less compliant to recommended prenatal care and suffer a higher risk of adverse perinatal outcomes. Several attempts to encourage optimal pregnancy follow-up have shown controversial results, particularly in high-income countries. Few studies have assessed financial incentives to encourage prenatal care, and none reported materno-fetal events as the primary outcome. Our study aims to determine whether financial incentives could improve pregnancy outcomes in women with low SES in a high-income country.

Methods and analysis This pragmatic

cluster-randomised clinical trial includes pregnant women with the following criteria: (1) age above 18 years, (2) first pregnancy visit before 26 weeks of gestation and (3) belonging to a socioeconomically disadvantaged group. The intervention consists in offering financial incentives conditional on attending scheduled pregnancy follow-up consultations. Clusters are 2-month periods with random turnover across centres. A composite outcome of maternal and neonatal morbidity and mortality is the primary endpoint. Secondary endpoints include maternal or neonatal outcomes assessed separately, qualitative assessment of the perception of the intervention and cost-effectiveness analysis for which children will be followed to the end of their first year through the French health insurance database. The study started in June 2016, and based on an expected decrease in the primary endpoint from $18 \%$ to $14 \%$ in the intervention group, we plan to include 2000 women in each group.

Ethics and dissemination Ethics approval was first gained on 28 September 2014. An independent

\section{Strengths and limitations of this study}

- Inadequate prenatal care has been associated with poor pregnancy outcomes, and in high-income countries, socioeconomically disadvantaged women have increased rates of both unattended pregnancy follow-up visits and pregnancy-related adverse outcomes. Among interventions that aim to promote adequate prenatal care, financial incentives have the potential to overcome some of the barriers, but data on the reality and the magnitude of such an effect on pregnancy outcomes are sparse.

- A cluster-randomised clinical trial, using a Zelen design, will run in about 40 public maternity units all over France, with the aim to include a total of 4000 women. The intervention group will receive a $€ 30$ incentive for each scheduled prenatal visit they have attended. The control group will be followed according to the national recommendations and local practices.

- The main outcome is a composite of maternal and neonatal adverse outcomes. Secondary outcomes will include qualitative assessments and the costeffectiveness of intervention.

- The definition of the low socioeconomic group relies on the type of healthcare insurance alone; no individual assessment will be conducted.

- Follow-up of children until their first birthday will only rely on electronic data from the French Health Insurance database.

data security and monitoring committee has been established. Results of the main trial and each of the secondary analyses will be submitted for publication in a peer-reviewed journal.

Trial registration number NCT02402855; pre-results. 


\section{INTRODUCTION}

\section{Background and rationale}

Prenatal care, that is, the medical and nursing care recommended during pregnancy, aims to prevent, if possible, any potential problems or to detect them early to organise appropriate management. A number of studies have demonstrated a relationship between fewer prenatal visits and poorer pregnancy outcomes. A large retrospective cohort in the USA demonstrated that women with inadequate prenatal care (defined as attending fewer than $50 \%$ of recommended visits) had an increased risk of preterm birth (OR 2.0, 95\% CI 1.9 to 2.0), low birth weight (LBW) (OR 1.7, 95\% CI 1.6 to 1.7 ) and infant mortality (OR 1.5, $95 \%$ CI 1.3 to 1.7 ) compared with women who received adequate prenatal follow-up. ${ }^{1}$ This association is even stronger in European cohort studies, even after adjusting for confounding factors. ${ }^{2-4}$

As the provision of prenatal care to pregnant women appears to be an effective way to improve perinatal outcomes, many countries have implemented specific recommendations for this care, with various expectations in different countries, the minimum being eight antenatal contacts, as recommended by WHO. ${ }^{5}$

Appropriate pregnancy follow-up is based on early initial access to a health professional and adequate compliance with the scheduled follow-up visits. It has been suggested that initial access is influenced by late recognition of the pregnancy and subsequent denial or acceptance, whereas follow-up depends on a strategy of weighing up and balancing out the perceived gains and losses. Personal resources in terms of time, money and social support as well as the services available are all taken into account. ${ }^{6}$

Perinatal outcomes in socioeconomically disadvantaged populations have been shown to be worse than those in the rest of the population, in terms of perinatal death, ${ }^{78}$ prematurity, ${ }^{9}{ }^{10}$ congenital anomalies and LBW. ${ }^{11-13}$ Access to prenatal care is insufficient in this at-risk population. This has been demonstrated in countries where access to care is not equal, such as the USA, as well as in countries such as Belgium or France, where free healthcare is provided for socially disadvantaged populations. ${ }^{14} 15$ In France, for example, the preliminary results of the preCARE cohort, which analysed data of about 10000 women, suggested that women covered with insurance for low-income people (Couverture Maladie Universelle (CMU) or Universal Medical Coverage) and insurance for illegal immigrants (Aide Médicale d'Etat (AME) or State Medical Assistance)) have, respectively, a $50 \%$ and $80 \%$ increased risk of severe maternal morbidity (relative risk of $1.5 ; 95 \%$ CI 0.95 to 2.3 and 1.8; 95\% CI 1.1 to 3.1, for CMU and AME women, respectively). ${ }^{4}$

In addition, adequate prenatal care has been shown to be cost-effective. This effect is largely due to the extremely high cost of care for preterm and LBW infants, with costs standing at over US\$100000 for extreme prematurity, between US\$40000 and US\$100 000 for early prematurity, between US $\$ 10000$ and US $\$ 30000$ for moderate prematurity and below US $\$ 4500$ for late prematurity. ${ }^{16}$

Several interventions to improve pregnancy follow-up have been assessed, and most of the recent literature on this topic has come from low-income and middle-income countries. In a systematic review published in 2016, Lassi and colleagues $^{17}$ assessed human resources for health (HRH) and showed that HRH interventions could contribute positively to the health worker's performance and thus improve maternal outcomes. The meta-analysis published in 2015 by the same authors suggested that community-based intervention packages could lead to a significant reduction in neonatal mortality (risk ratio (RR) $0.75,95 \%$ CI 0.67 to 0.83 ) and a possible effect on maternal mortality (RR 0.80 ; $95 \%$ CI 0.64 to 1.00$){ }^{18}$

In the few studies conducted in high-income countries recently, several reinforcement interventions have been tested. A study conducted in the USA among primiparous African-American women assessed four levels of incremental interventions, which combined financial support with enhanced postdelivery follow-up. ${ }^{19}$ None of these interventions proved to be effective, even as a numerical trend, in reducing premature delivery or the proportion of small for gestational age (SGA) infants.

More recently, a study conducted in Ireland, among women from a disadvantaged community in Dublin, showed that a home-visit programme had no impact on any of the neonatal outcomes. ${ }^{20}$ In contrast, ${ }^{20} \mathrm{a}$ meta-analysis published in 2016 by Sandall and colleagues suggested that women who received midwife-led continuity models of care were less likely to experience poor pregnancy outcomes than women who received other models of care. $^{21}$

Some studies have suggested that the use of incentives may improve prenatal care and neonatal outcomes. A retrospective cohort study in Canada demonstrated that low-income pregnant women who participated in a prenatal support programme beginning early in pregnancy (before 21 weeks of gestation) had significantly decreased rates of LBW infants compared with women who enrolled later in pregnancy (after 30 weeks of gestation) (RR $0.47,95 \%$ CI 0.22 to 0.98$){ }^{22}$

A Cochrane review on this topic published in $2015^{23}$ was only able to show that pregnant women receiving incentives were more likely to obtain adequate quality prenatal care (mean difference 5.84, 95\% CI 1.88 to 9.80) as none of the included trials reported on preterm birth, SGA and perinatal deaths. This Cochrane review concluded that data from randomised trials, powered to show results on maternal and neonatal outcome, are urgently needed to inform public health authorities. Although it has been suggested that incentive programmes are effective, for example, for smoking cessation during pregnancy, ${ }^{24}$ this approach remains highly controversial. ${ }^{25}$

This paper describes the protocol of an adequately powered cluster-randomised controlled trial to explore the effectiveness on perinatal outcomes of financial 
incentives linked to the use of prenatal care and targeting socioeconomically disadvantaged women.

\section{Objectives}

Primary objectives

The primary objective is to assess the superiority of a conditional cash transfer programme in addition to the best standard of care compared with the best standard of care alone among socioeconomically disadvantaged women. The best standard of care is based on the guidelines defined by French health authorities (Haute Autorité de santé (HAS)), and superiority will be assessed in terms of maternal-fetal outcomes.

\section{Secondary objectives}

1. To determine whether a conditional cash transfer programme can improve neonatal outcomes.

2. To determine whether a conditional cash transfer programme can improve maternal pregnancy outcomes.

3. To assess through a qualitative approach, among women of the incentive programme group, their acceptance of the programme and whether it has a significant impact on their adherence to prenatal care.

4. To assess, among women from both groups, determinants of inadequate prenatal care.

5. To assess the cost-effectiveness and budget impact of the conditional cash transfer programme compared with the best standard of care without the conditional cash transfer.

\section{Trial design}

The NAITRE study is a pragmatic multicentre, open-label cluster-randomised trial using a parallel arm design.

\section{METHODS: PARTICIPANTS, INTERVENTIONS, OUTCOMES Study setting}

During year 1 of the study, June 2016 to June 2017, 10 centres, all tertiary obstetric departments, included women. These centres are located throughout France. Starting in June 2017, the study will be extended to about 30 additional primary to tertiary obstetric departments, assuming that the qualitative study shows no negative feeling of the women (see specific section below).

The cluster randomisation procedure was chosen in order to allow a Zelen design, ${ }^{26}$ that is, women being randomised before they consent to participate and only receiving information for the group they will be allocated to. This is of particular relevance as, when patients do not receive their preferred treatment in randomised trials, there may be difficulties with patient recruitment and scientific problems with bias. Centres, and not women, are being randomised. To account for heterogeneity among centres and the relatively small number of centres, about 40, clusters are 2-month periods with random turnover across centres, meaning that each centre will contribute both to the intervention and the control groups.

Patient enrolment started in June 2016 and is expected to end in December 2019.

Figure 1 summarises the design of the trial, and each aspect of the trial is described in detail below.

\section{Eligibility criteria}

The study includes pregnant women: (1) above the age of 18 , (2) with their first pregnancy visit in one of the participating centres before the end of the 26th week of amenorrhoea and (3) with social insurance for low-income people (CMU) or illegal immigrant status (AME).

The only non-inclusion criteria are: (1) women not able to understand the study and (2) women under legal supervision. There are no other restrictions to the inclusion criteria as the study has a pragmatic concept.

\section{Interventions \\ Control group}

Women in the control group receive prenatal care according to the standard practice, as set out in national guidelines. Women's personal medical history and pregnancy medical data are collected up to 1 month

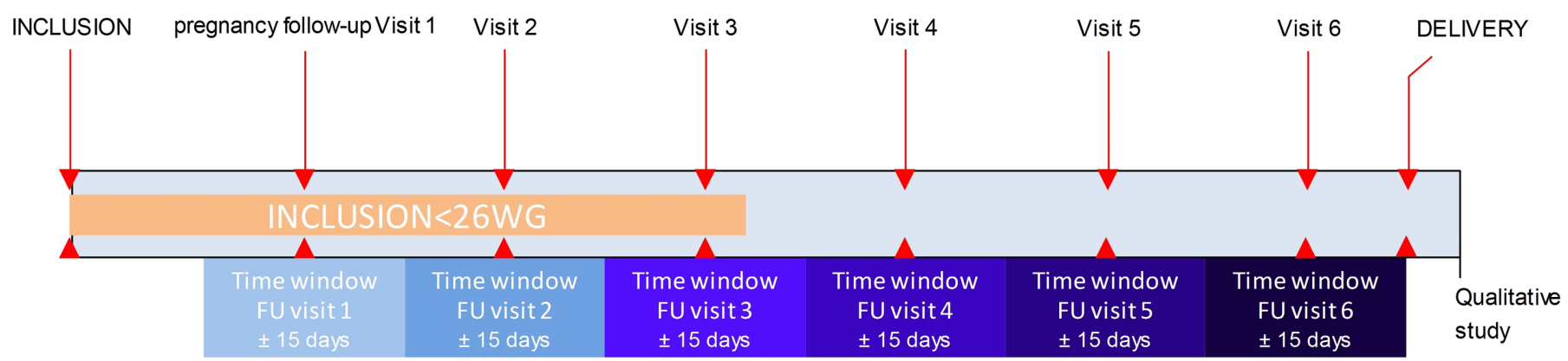

Figure 1 Scheduled prenatal care visits and time window for financial incentives. If a woman shows up for a scheduled visit more than 2 weeks after her standard preplanned visit, she will receive the incentive for the next visit. This means that she will not attend a scheduled visit without receiving an incentive (with the limit of no more than one incentivised visit per month), but she may receive fewer incentive payments than the maximum she could have obtained. 26WG, 26 weeks of gestation; FU, follow-up. 
postdischarge after delivery, whereas data for their child will be collected up to their first birthday.

\section{Intervention group}

Women in the intervention group are given a payment card on their first visit, the inclusion visit. The card will be credited with $€ 30$ after each scheduled prenatal visit, with a maximum of one $€ 30$ incentive per month. Cash transfer starts at the first scheduled visit after the inclusion visit.

No additional incentives will be provided if more visits are deemed necessary because of a specific pregnancy follow-up programme or unplanned visits to the emergency department. Inclusion using the eCRF (electronic Case Report Form) automatically generates the scheduled prenatal visits programme according to the national guidelines. Figure 1 shows the agenda for prenatal visits with time windows for incentives.

\section{Outcomes}

\section{Primary outcome}

The primary endpoint is a global composite endpoint of perinatal morbidity and mortality, defined as the occurrence of at least one complication of pregnancy, whether maternal, fetal or neonatal.

- For infants: perinatal death, premature birth (before 37 weeks of amenorrhoea), intrauterine growth restriction (estimated weight $<$ the 10th percentile for gestational age and abdominal circumference below the 2.5 th percentile), LBW $(<2500 \mathrm{~g}$ at term), early neonatal encephalopathy (asphyxia at birth) and cerebral anomalies of neonatal or perinatal origin (anoxic or ischaemic encephalopathy, periventricular leukomalacia or intraventricular haemorrhage), lesion of the brachial plexus (clinical diagnosis) and probable or certain maternal-fetal infection (clinical and biological diagnosis). Birth of a child with a congenital abnormality is considered a failure if it results from a lack of prenatal diagnosis, but a success if it results from parental choice. Therapeutic interruptions of pregnancy for congenital malformation diagnosed during pregnancy are likewise considered part of the optimal follow-up and are counted as successes.

- For the mother: maternal death, phlebitis, pulmonary embolism (diagnosed by Doppler ultrasound of the lower limbs and/or spiral scan and/or pulmonary scintigraphy), pre-eclampsia or eclampsia, postpartum haemorrhage requiring transfusion, conservative surgery or embolisation of uterine arteries or hysterectomy, postpartum endometritis (clinical diagnosis) and third-degree or fourth-degree perineal lesion with anal sphincter injury.

Perinatal outcomes will be assessed at hospital discharge of both the mother and the infant (last discharged).
Secondary outcomes

1. Adverse perinatal outcome in the child, as previously defined.

2. Adverse perinatal outcome in the mother, as previously defined.

3. Number of prenatal consultations following the French standard care recommendations. All outpatient visits, including those prospectively scheduled by the study protocol, will be retrieved using the French Health Insurance database (Système National d'Informations Inter Régimes de l'Assurance Maladie (SNIIR-AM)).

4. Qualitative assessment of barriers to adequate prenatal follow-up and the perception of the intervention by some of the participating women and health professionals.

5. Differential cost-effectiveness ratio associated with financial incentive versus no compensation and expressed in terms of cost per complication avoided.

6. Overall budget impact.

\section{Participant timelines}

Women will be followed according to the official antenatal care programme and to requests from their obstetrician. No additional visits or tests of any type will be requested because of their participation in the trial. Data will be recorded from inclusion to discharge from the maternity ward.

Newborns will be followed up to discharge from hospital.

For the cost-effectiveness study, data on health resources utilisation, that is, outpatient clinics, blood or imaging tests performed and medication purchased, will be collected through the national health insurance database, up to the first year of age for the children.

Figure 2 shows the overall management of women included in the study according to the randomisation group.

\section{Sample size}

According to the 2010 French perinatal survey, the estimated prevalence of adverse pregnancy outcomes, according to our primary outcome definition, is estimated at $12 \%$ in the general population and $18 \%$ in socioeconomically disadvantaged women. ${ }^{27}$ The hypothesis supporting our sample size calculation is that the financial incentive, by increasing adherence to prenatal care, can reduce this difference by two-thirds, thus resulting in a rate of complicated pregnancies in the intervention group of $14 \%$. To ensure a power of $80 \%$ and a significance level of $0.05,1314$ women per group are needed.

In order to obtain accurate data, the frequency of randomisation was set at every 2 months. According to the randomisation procedure, we will have 420 clusters (2-month periods over 3 years for the 10 participating maternity departments of the first phase and over 2 years in the up to 30 additional centres included in the second phase) and about eight women per cluster. 


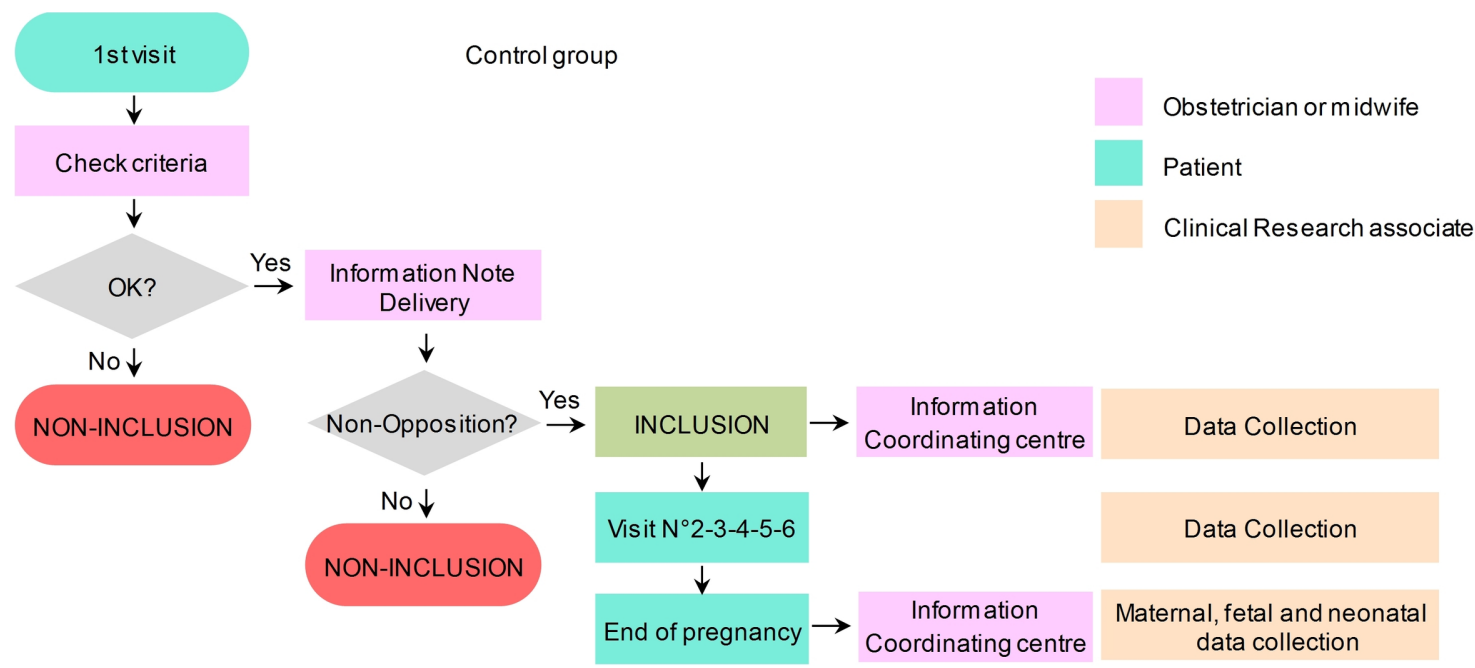

We hypothesised an intracluster correlation coefficient (ICC) of 0.01 , meaning that 1526 women are necessary. ${ }^{28}$ Allowing for $20 \%$ attrition, 1900 women per group are needed, and we set the sample size at 2000 per group. An ICC of 0.01 was selected because the trial assesses an intervention aimed directly at the patient and an outcome measurement for which the variance between practices is low compared with the variability between patients within a practice.

\section{Recruitment}

All eligible women registering for maternity care at the trial sites during the study period will be invited to participate in the study. The list of the participating centres can be found on ClinicalTrials.gov (NCT02402855).

Information sheet and consent form for the intervention group (online supplementary annex 1) and the control group (online supplementary annex 2 ) are available in
18 languages. The self-administered deprivation questionnaire embedded in both forms is available in French only.

\section{Cost-effectiveness analysis}

Study design

A cost-effectiveness analysis comparing the financial incentive strategy associated with prenatal care of socioeconomically disadvantaged women versus no financial incentive will be carried out. The reference strategy is standard prenatal care in the absence of financial incentives.

This analysis will be conducted from a societal perspective in such a way as to favour a sufficiently broad perspective to take into account all stakeholders involved in the intervention.

\section{Outcomes}

Efficacy is a composite criterion that includes complications of the child during his/her first year of life (such 
as perinatal death, prematurity or neurological impairment) and maternal adverse events (including postpartum haemorrhage, endometritis). Direct costs will be considered from a societal perspective. The effectiveness of the intervention will be expressed in terms of cost per complication avoided. The time horizon of the study will be at most 1 year and 9 months, corresponding to the period of pregnancy and a 1-year postdelivery follow-up period. Given the relatively short duration of follow-up, costs and effectiveness will not be updated. In addition to data collected in the CRF (CAse Report Form), all direct medical costs up to 1 year postdelivery will be retrieved from the National Health Insurance database.

\section{Qualitative assessment method}

\section{Practical aspects}

Women will be selected after delivery according to their medical follow-up during pregnancy.

Every woman included in the NAITRE study is eligible for the qualitative study except in case of stillbirth, postnatal death or if the baby suffers from a seriously condition. As the women should be in position to relate their pregnancy, it seems it would be difficult for women in mourning.

To ensure adequate representativeness, women will be selected in both the intervention and control groups to obtain different ages, primiparous and multiparous women, different economic conditions and different adherence to prenatal care. This information is given by investigators after the delivery. Semidirected individual interviews will aim to understand the determinants of medical follow-up during pregnancy. The principle is to get the respondents to describe situations that highlight their habits, their social representations or their emotions. Women will be asked to describe their health practices during pregnancy, what was important for their medical follow-up and what led them to renounce scheduled care. For women who received the financial incentive, it is also a question of asking them how they experienced this incentive and what it may or may not have brought them.

In order to get this information, a retrospective study will be conducted. Interviews will take place within 1 month of delivery. Women will be invited to return to the referral centre or will be seen at their home (with a specific incentive of $€ 40$ credited to the card for those in the intervention group or a payment card for those in the control group).

The method of semistructured individual interviewing is based on an interview guide containing closed or open questions, designed so as not to be inductive with regard to the themes to be explored. Interviews will begin with an open-ended question: "Can you tell me about your pregnancy?"

Depending on the construction of the interviewee's story and according to the logic of a dialogue, the questions of the interview guide will be asked only if the information has not been provided spontaneously. These questions will be grouped in an interview guide available to the interviewer at the time of the interview with the respondent. These interview guides are constructed in such a way so as to favour a more or less long interview depending on the interviewee (about 1hour), using simple, easily understandable questions.

Whenever necessary, the interview will be conducted with the assistance of an interpreter (telephone interpreter, planned budget).

Interview grid for the qualitative survey is shown, in French language only, in online supplementary annex 3.

\section{Method of collection}

The respondents will be met individually in a confidential location. Interviews will be conducted by a social scientist trained in qualitative research. Women will be asked to allow the interview to be recorded, transcribed and analysed anonymously.

\section{METHODS: ASSIGNMENT OF INTERVENTION Allocation}

Centres are randomly allocated to 2-month periods of either the intervention or control group. Randomisation is centrally generated, and before the end of a period, centres are not aware of the group they will be allocated to for the next period, in order to prevent the postponement of inclusions at the end of one control period so as to include women in the next intervention period.

The inclusion of women and the reporting of further pregnancy follow-up visits are made through a web-based eCRF (www.etudenaitre.fr), where the information sheet and consent form, translated in 18 foreign languages, can be downloaded directly. Women will be included prospectively by obstetricians and midwives in the participating centres, based on their type of health insurance coverage.

\section{Blinding}

By the nature of the study, blinding of the intervention is not feasible. However, all analyses will be conducted by a statistician blinded to allocation to the intervention or control group.

\section{METHODS: DATA COLLECTION MANAGEMENT AND ANALYSIS Data collection methods}

Data will be collected through the eCRF. Inclusion and non-inclusion criteria, as well as attendance at each scheduled visit in the intervention group, will be prospectively entered into the eCRF. All other data, for maternal and neonatal outcomes, will be extracted from individual pregnancy records, in each of the participating centres, and collected into the eCRF by clinical research assistants.

For the cost-effectiveness study, data for the first year of life will be extracted from the French Hospitalisation (Le Programme de Médicalisation des Systèmes d'Information) and Health Insurance (SNIIR-AM) databases and analysed at Dijon University Health Centre. 


\section{Data management}

The data will be managed by the Clinical Investigation Centre (CIC INSERM 1432) at Dijon University Hospital.

\section{Statistical method}

Descriptive analysis

Clinical and demographic characteristics of the women will be presented, and the comparability of the two groups of women regarding pregnancy risk factors will be assessed.

\section{Primary outcome analysis}

The primary criterion, as described previously, is binary. The principal analysis will be a proportion comparison using the $\chi^{2}$ test adjusted for the design effect, on an individual basis.

As the cluster randomisation will generate a lower quality of balance between covariates, we will additionally study the following parameters, which potentially interfere with maternal or fetal outcomes, using univariate logistic regression:

- type of centre (local/intermediate-risk/high-risk maternity unit)

- proportion of deprived women in the centre (three levels defined as $<25$ th, 25th-75th and $>75$ th percentile of the observed proportions in the different centres)

- age $(<20,20-35,>35$ years old $)$

- parity $(0,1$ or 2 , more than 2$)$

- type of free healthcare (CMU Protection Complémentaire/AME).

Interactions of these parameters and the effect of the intervention will be searched for using subgroup analyses.

A multivariable multilevel logistic regression adjusted for all the covariates with a $\mathrm{p}<0.20$ will then be performed, taking into account the eventual interactions. A random-effects term referring to clusters will be included in the model. The multivariate building process will use a stepwise approach. For each step, the Akaike information criterion (AIC) scores will be calculated to measure the relative goodness of fit of the different models, where the lower the AIC, the better the fit. The model with the lowest AIC score will be retained for the analyses. $^{29}$

\section{Secondary outcome analysis}

1. Adverse outcomes in the child: same as the principal analysis, using this outcome.

2. Adverse outcomes in the mother: same as the principal analysis, using this outcome.

3. The number of prenatal consultations following the HAS standard care recommendations will be analysed using weighted means taking into account the cluster randomisation.

An intermediate analysis will be performed after the first phase, but using a significance level of 0.0001 in order not to impair the study power.
Significance threshold

The results will be considered statistically significant if $\mathrm{p}<0.05$.

\section{Statistical software}

The analyses will be performed using SAS software, latest version at the time of analysis. Statisticians will be blinded to the study groups.

\section{Analysis of qualitative survey}

Raw data are analysed according to a thematic analysis method. From this progressive work of conceptualisation and continuous comparison of the different situations, a theoretical system responding to our problems will be defined. A triangulation of data will be done as two sociologists will code the interview transcriptions. Nvivo software, which allows the creation of a code and has been well validated for qualitative analyses, will be used. ${ }^{30}$ Qualitative data will be analysed during the data collection. The collection of data will continue until concept saturation is reached. Theoretical saturation will be assessed. ${ }^{31}$ Practically, saturation is reached when no new data could add more information to meet the research objectives. Data will be analysed by two researchers, and the collection of data will end when two consecutive interviews have not generated any new information.

\section{Statistical analysis of cost effectiveness}

A differential cost-effectiveness ratio associated with the intervention versus no intervention will be calculated by comparing the difference in average costs with the difference in average efficiencies.

In order to test the robustness of the conclusions drawn, sensitivity analyses will be carried out on the parameters likely to have an influence on the results.

A non-parametric bootstrap analysis of the cost and efficiency differential observed between the two strategies will allow uncertainty to be taken into account and to estimate $95 \%$ CIs for the differential ratios of the baseline analysis.

\section{METHODS: MONITORING Monitoring}

As the study has been deemed non-interventional by the ethics committee, legally no formal monitoring is requested. Nonetheless, the study will be monitored for quality and regulatory compliance. The monitoring will be supervised by the promoter (Dijon-Bourgogne University Hospital) in all of the participating centres. The frequency depends on inclusion rates, questions and pending issues from earlier audits: once or twice a year.

\section{Harm}

Steering and data and safety monitoring committees

The coordinating centre at Centre Hospitalier Universitaire Dijon-Bourgogne, Centre d'Investigations Cliniques (CIC INSERM 1432), takes responsibility for all aspects 
of the study: ethical, regulatory, study conduction, data management and publication strategy.

The steering committee is composed of the principal investigators of the first 10 centres, one representative from each perinatal care network involved in the study, a midwife, a social worker and a methodologist. A phone meeting is held every 4 months. The role of the steering committee is to ensure that the study is running according to study protocol and to share solutions to overcome difficulties in recruiting participants.

The data monitoring and safety committee (DSMC) is composed of two obstetricians, who specifically take care of socioeconomically disadvantaged women, a methodologist, an ethics specialist and a paediatrician. DSMC will essentially review results of the qualitative survey conducted during the first year and follow observed versus expected recruitment curves. If the qualitative survey were to suggest that the intervention stigmatises women, the scientific committee will have the right to stop the study. As women will be managed according to French guidelines during their antenatal and postnatal period and will thus not be affected by the study, no harm other than potential stigmatisation is expected. No interim analysis has been planned, as it would have had a significant impact on sample size.

\section{Auditing}

The study has been deemed non-interventional by the ethics committee; as a result, by law no formal auditing will be conducted.

\section{Ethics and dissemination}

Research ethics approval

Ethics approval was given by the Dijon Ethics Committee (CPP Est-1) on 18 September 2014, and the protocol was amended thereafter.

The study obtained funding from the French Ministry for Health in December 2014 (PREPS-14-0173) and the study is promoted by Dijon Bourgogne University Hospital (France).

Authorisation for holding the computerised databases was granted on 21 March 2016 by CNIL. This long delay was due to conflict between the reasoning of CNIL, for which the preservation of private life is its top priority, and the French source of public funding, which needs to know precisely to whom public money, in this case the financial incentive, is being given.

It was registered in ClinicalTrials.gov with the identifier NCT02402855, on 17 March 2015, at the French Medicines Agency with identifier 2014-A01319-38 and at CNIL with request for authorisation number 915385 .

\section{Protocol amendments}

The study is currently running according to the fifth amended version, 30 June 2016.

\section{Consent or assent}

The study is non-interventional according to the first approved version of the protocol; no formal consent is required to be recruited.
Formal consent, from the women only, is requested for the collection of data concerning their children (see information sheet in online supplementary appendices).

\section{Ancillary and post-trial care}

As the study does not affect the usual care of women, no post-trial care has been scheduled. No ancillary studies have been planned so far.

\section{Dissemination policy}

A manuscript with the results of the primary study will be published in a peer-reviewed journal. Separate manuscripts will be written on each of the secondary aims, and these will also be submitted for publication in peer-reviewed journals.

The results will be presented at scientific meetings, and specific communication will be organised to target health professionals, policy decision-makers, regulatory bodies and women.

Recruitment is ongoing; the first women were recruited on 4 June 2016 in the control group and on 10 June 2016 in the intervention group.

\section{DISCUSSION}

This will be the first study to provide rigorous evidence regarding the effectiveness of financial incentives to improve the use of prenatal care and their impact on perinatal outcomes for the mother and the fetus. A recent Cochrane meta-analysis ${ }^{23}$ emphasised the need for such an assessment, and it is of particular interest to note that no such studies have been carried in Europe so far.

The conditional cash transfer is not supposed to replace any other public health policies aimed to promote appropriate prenatal care, but it may help to overcome some of the barriers that stand in the way of adequate follow-up. Most of the studies have been conducted in low-income countries, where, besides many other factors, the structure of the healthcare system and healthcare facilities are very different from those in developed countries.

For example, in 2005, the Indian Government implemented the Janani Suraksha Yojana programme, which incentivises poor women to give birth in a health facility by providing them with a cash transfer on discharge. There was, however, no formal assessment of the efficacy. Whereas this programme was associated with a very significant increase in the rate of delivery in healthcare facilities (from $39 \%$ to $74 \%$ ), a recent survey suggested that beyond the cash incentive, the shift in the social norm and the women's own perception played a major role in this effect. ${ }^{32}$ Interestingly, this programme was shown to be associated with a reduction in neonatal and perinatal deaths. ${ }^{33}$

Financial incentive programmes to promote smoking cessation during pregnancy have been assessed and have recently been reported to be associated with a reduced likelihood of LBW. ${ }^{34}$ 
If our intervention proves to be effective, its implementation may have to overcome several difficulties, including tailoring the intervention to women most likely to respond, increasing acceptability of the concept among public bodies and health professionals and proving the intervention to be cost-effective. It has been suggested that, for some interventions, efforts have to be made to increase acceptability, even when they have been proven to be effective. ${ }^{35}$ All of these aspects will be covered by our research programme.

\section{Author affiliations}

${ }^{1}$ Centre d'Investigation Clinique INSERM 1432, Centre Hospitalier Universitaire de Dijon, Dijon, Bourgogne, France

${ }^{2}$ Centre de Recherche INSERM LNC-UMR1231, UFR Sciences Santé, Dijon, France

${ }^{3}$ Université Bourgogne-Franche Comté, Dijon, France

${ }^{4}$ Centre de Recherche en Economie Statistique (CREST), Malakoff, France

${ }^{5}$ Unité de Soutien Méthodologique à la Recherche, CHU Dijon-Bourgogne, Dijon, Bourgogne, France

${ }^{6}$ Neurosciences Intégratives et cliniques EA 481, Université Bourgogne FrancheComté, Besançon, France

7"Ethique et Progrès médical", CIC INSERM 1431, Centre Hospitalier et Universitaire de Besançon, Besançon, France

${ }^{8}$ Service de Gynécologie Obstétrique, CHU de Besancon, Paris, France

${ }^{9}$ Service de Gynécologie Obstétrique, CHU de Bicetre, Paris, France

${ }^{10}$ Service de Gynécologie Obstétrique, CHU de Brest, Brest, France

${ }^{11}$ Service de Gynécologie Obstétrique, CHRU De Lille, Lille, France

${ }^{12}$ Service de Gynécologie Obstétrique, Hospices Civils de Lyon—Hôpital Femme

Mère Enfant, Lyon, Rhône-Alpes, France

${ }^{13}$ Service de Gynécologie Obstétrique, CHU de Marseille Hôpital Nord, Marseille, Provence-Alpes-Côte d'Azu, France

${ }^{14}$ Service de Gynécologie Obstétrique, CHU Robert Debré, Paris, Île-de-France, France

${ }^{15}$ Service de Gynécologie Obstétrique, CHU de Saint Etienne, Saint Etienne, France

${ }^{16}$ Service de Gynécologie Obstétrique, CHU de Toulouse, Toulouse, Midi-Pyrénées, France

${ }^{17}$ Service de Gynécologie Obstétrique, CHU Bretonneau, Tours, France

${ }^{18}$ Service de Gynécologie Obstétrique, Centre Hospitalier de Saint Denis, Saint Denis, Île-de-France, France

${ }^{19}$ INSERM UMR 1153, Obstetrical, Perinatal and Pediatric Epidemiology Research Team (EPOPé), Center for Epidemiology and Statistics Sorbonne Paris Cité, DHU Risks in Pregnancy, Paris Descartes University, Paris France, Paris, France

${ }^{20}$ Department of Economics, Massachusetts Institute of Technology, Cambridge,

Massachusetts, USA

${ }^{21}$ Service de Gynécologie Obstétrique, CHRU Strasbourg, Strasbourg, Alsace, France

Collaborators NAITRE study group members: CHU Besançon: Professor Didier Riethmuller, Dr Aude Bourtembourg, Dr Nicolas Mottet, Dr Claire Toubin, Guillaume Boiteux. CHU Bicêtre: Professor Marie-Victoire Senat, Dr Marie Houlier, Dr MarieEmmanuelle Neveu, Solène Farbre. CHU Brest: Philippe Merviel. CHU Lille: Eliane Catteau, Professor Dominique Deplanque. CHU Lyon: Nathalie Laurenceau, Anne Leroux. CHU Marseille, Hôpital Nord: Julie Blanc, Valérie Vaugirard, Muriel Athlani, Sandrine Pettazzoni, Nathalie Lesavre. CHU Robert Debré: Lauriane Nikel, Elodie Gustave, Professor Jacqz-Aigrin. CHU Saint Etienne: Professor Celine Chauleur, Dr Andréa Buchmuller. CHU Toulouse: Professor Christophe Vayssiere. CHU Tours: Anne Viallon, Catherine Guerin, Dr Caroline Diguisto, Dr Stephanie Chretien, Dr Christelle Denis, Dr Carine Arlicot, Dr Jerome Potin, Dr Nathalie Trignol-Viguier.

Contributors Study design: MB, BC, A-CB, AG-M, ED and ILR. Obtaining funding and drafting publication: $\mathrm{MB}$. Reviewing and amendment of the protocol, reviewing of the paper: AE-L, ET, FF, PD, MD, XC-T, TS, TB, MM, FP, GH, CD-T, IF, LL and NM-B. Authorship eligibility guidelines and any intended use of professional writers: $\mathrm{MB}$ will be first author of the main publication of study results, he will be last author of affiliated papers, if any, such as results of the qualitative and the cost effectiveness surveys. AG-M will be first author of the qualitative survey and A-CB of the costeffectiveness study. MB will sign as last author. One investigator will be listed as an author in each of the participating centres, with the investigator of the best recruiting centre (based on the number of participants recruited pro-rata with the time spent by the centre on the study and the number of pregnancies followed during that time). We do not intend to use a professional writer.

Funding The study has been funded by the Ministère de la Santé, Direction Générale de l'Offre de Soins (grant number PREPS-14-0173). Sponsor: CHU DijonBourgogne, Délégation à la Recherche Clinique et à l'Innovation. Sponsor Contact Person: Catherine Renaud, tel + 333802931 53; catherine.renaud@chu-dijon.fr.

Disclaimer Neither funder nor sponsor had any role in the study design, writing of this report or the decision to submit the report for publication.

\section{Competing interests None declared.}

Ethics approval The study was approved by a central ethics committee (Comité de Protection des Personnes Est-1), by the French Medicines Agency, and data collection and recording was approved by the CNIL (Commission Nationale de I'Informatique et des libertés).

Provenance and peer review Not commissioned; externally peer reviewed.

Open Access This is an Open Access article distributed in accordance with the Creative Commons Attribution Non Commercial (CC BY-NC 4.0) license, which permits others to distribute, remix, adapt, build upon this work non-commercially, and license their derivative works on different terms, provided the original work is properly cited and the use is non-commercial. See: http://creativecommons.org/ licenses/by-nc/4.0/

(c) Article author(s) (or their employer(s) unless otherwise stated in the text of the article) 2017. All rights reserved. No commercial use is permitted unless otherwise expressly granted.

\section{REFERENCES}

1. Cox RG, Zhang L, Zotti ME, et al. Prenatal care utilization in Mississippi: racial disparities and implications for unfavorable birth outcomes. Matern Child Health J 2011;15:931-42.

2. Raatikainen K, Heiskanen N, Heinonen S. Under-attending free antenatal care is associated with adverse pregnancy outcomes. BMC Public Health 2007;7:268.

3. Blondel B, Marshall B. Poor antenatal care in 20 French districts: risk factors and pregnancy outcome. J Epidemiol Community Health 1998;52:501-6.

4. Linard M, Deneux-Tharaux C, Azria E, et al. Association between inadequate antenatal care utilisation and severe perinatal and maternal morbidity: an analysis in the PreCARE cohort. BJOG 2017:doi: 10.1111/1471-0528.14819. [Epub ahead of print 29 Aug 2017].

5. World Health Organization. WHO recommendations on antenatal care for a positive pregnancy experience. Geneva, Switzerland: WHO, 2016. http://appswhoint/iris/bitstre am/10665/250796/1/9789241549912-engpdf?ua=1 (accessed $12 \mathrm{Jul}$ 2017).

6. Downe S, Finlayson $\mathrm{K}$, Walsh $\mathrm{D}$, et al. 'Weighing up and balancing out': a meta-synthesis of barriers to antenatal care for marginalised women in high-income countries. BJOG 2009;116:518-29.

7. Racape J, Schoenborn C, Sow M, et al. Are all immigrant mothers really at risk of low birth weight and perinatal mortality? The crucial role of socio-economic status. BMC Pregnancy Childbirth 2016;16:75

8. Freemantle N, Wood J, Griffin C, et al. What factors predict differences in infant and perinatal mortality in primary care trusts in England? A prognostic model. BMJ 2009;339:b2892.

9. Wood S, McNeil D, Yee W, et al. Neighbourhood socio-economic status and spontaneous premature birth in Alberta. Can J Public Health 2014;105:383-8.

10. Smith LK, Draper ES, Manktelow BN, et al. Socioeconomic inequalities in very preterm birth rates. Arch Dis Child Fetal Neonatal Ed 2007;92:F11-4

11. Valero De Bernabé J, Soriano T, Albaladejo R, et al. Risk factors for low birth weight: a review. Eur J Obstet Gynecol Reprod Biol 2004;116:3-15.

12. Maness SB, Buhi ER. Associations between social determinants of health and pregnancy among young people: a systematic review of research published during the past 25 years. Public Health Rep 2016;131:86-99.

13. Lorch SA, Enlow E. The role of social determinants in explaining racial/ethnic disparities in perinatal outcomes. Pediatr Res 2016;79:141-7.

14. Fobelets M, Beeckman K, Hoogewys A, et al. Predictors of late initiation for prenatal care in a metropolitan region in Belgium. A cohort study. Public Health 2015;129:648-54. 
15. Gonthier C, Estellat C, Deneux-Tharaux C, et al. Association between maternal social deprivation and prenatal care utilization: the PreCARE cohort study. BMC Pregnancy Childbirth 2017;17:126.

16. Soilly AL, Lejeune C, Quantin C, et al. Economic analysis of the costs associated with prematurity from a literature review. Public Health 2014;128:43-62.

17. Lassi ZS, Musavi NB, Maliqi B, et al. Systematic review on human resources for health interventions to improve maternal health outcomes: evidence from low- and middle-income countries. Hum Resour Health 2016;14:10.

18. Lassi ZS, Bhutta ZA. Community-based intervention packages for reducing maternal and neonatal morbidity and mortality and improving neonatal outcomes. Cochrane Database Syst Rev 2015;3:CD007754.

19. Kitzman $\mathrm{H}$, Olds DL, Henderson $\mathrm{CR}$, et al. Effect of prenatal and infancy home visitation by nurses on pregnancy outcomes, childhood injuries, and repeated childbearing. A randomized controlled trial. JAMA 1997;278:644-52.

20. Doyle O, McGlanaghy E, Palamaro-Munsell E, et al. Home based educational intervention to improve perinatal outcomes for a disadvantaged community: a randomised control trial. Eur J Obstet Gynecol Reprod Biol 2014;180:162-7.

21. Sandall J, Soltani H, Gates S, et al. Midwife-led continuity models versus other models of care for childbearing women. Cochrane Database Syst Rev 2016;4:CD004667.

22. Canning PM, Frizzell LM, Courage ML. Birth outcomes associated with prenatal participation in a government support programme for mothers with low incomes. Child Care Health Dev 2010;36:225-31.

23. Till SR, Everetts D, Haas DM. Incentives for increasing prenatal care use by women in order to improve maternal and neonatal outcomes. Cochrane Database Syst Rev 2015;12:CD009916.

24. Tappin D, Bauld L, Purves D, et al. Financial incentives for smoking cessation in pregnancy: randomised controlled trial. $B M J$ 2015;350:h134.
25. Hoddinott P, Morgan H, MacLennan G, et al. Public acceptability of financial incentives for smoking cessation in pregnancy and breast feeding: a survey of the British public. BMJ Open 2014;4:e005524.

26. Zelen M. A new design for randomized clinical trials. N Engl J Med 1979;300:1242-5.

27. Blondel B, Lelong $\mathrm{N}$, Kermarrec $\mathrm{M}$, et al. Trends in perinatal health in France from 1995 to 2010. Results from the French National Perinatal Surveys. J Gynecol Obstet Biol Reprod 2012;41:e1-5.

28. Campbell MK, Thomson S, Ramsay CR, et al. Sample size calculator for cluster randomized trials. Comput Biol Med 2004;34:113-25.

29. Akaike H. A new look at the statistical model identification. IEEE Trans Automat Contr 1974;19:716-23.

30. Enright G, Gyani A, Raadsma S, et al. Evaluating factors influencing the delivery and outcomes of an incentive-based behaviour change strategy targeting child obesity: protocol for a qualitative process and impact evaluation. BMJ Open 2016;6:e012536.

31. Kerr C, Nixon A, Wild D. Assessing and demonstrating data saturation in qualitative inquiry supporting patient-reported outcomes research. Expert Rev Pharmacoecon Outcomes Res 2010;10:269-81.

32. Sidney $\mathrm{K}$, Tolhurst $\mathrm{R}$, Jehan $\mathrm{K}$, et al. 'The money is important but all women anyway go to hospital for childbirth nowadays' - a qualitative exploration of why women participate in a conditional cash transfer program to promote institutional deliveries in Madhya Pradesh, India. BMC Pregnancy Childbirth 2016;16:47.

33. Lim SS, Dandona L, Hoisington JA, et al. India's Janani Suraksha Yojana, a conditional cash transfer programme to increase births in health facilities: an impact evaluation. Lancet 2010;375:2009-23.

34. Zhang X, Devasia R, Czarnecki G, et al. Effects of incentive-based smoking cessation program for pregnant women on birth outcomes. Matern Child Health J 2017;21:745-51.

35. Wen X, Higgins ST, Xie C, et al. Improving public acceptability of using financial incentives for smoking cessation during pregnancy: a randomized controlled experiment. Nicotine Tob Res 2016;18:913-8. 